\title{
Force variability during isometric wrist flexion in highly skilled and sedentary individuals
}

\author{
Konstantinos Salonikidis • Ioannis G. Amiridis • \\ Nikolaos Oxyzoglou • Eduardo Saez Saez de Villareal • \\ Andreas Zafeiridis · Eleftherios Kellis
}

Accepted: 21 August 2009 / Published online: 16 September 2009

(C) The Author(s) 2009. This article is published with open access at Springerlink.com

\begin{abstract}
The association of expertness in specific motor activities with a higher ability to sustain a constant application of force, regardless of muscle length, has been hypothesized. Ten highly skilled (HS group) young tennis and handball athletes and 10 sedentary (S group) individuals performed maximal and submaximal $(5,10,20,50$, and $75 \%$ of the MVC) isometric wrist flexions on an isokinetic dynamometer (Kin-Com, Chattanooga). The wrist joint was fixed at five different angles $(230,210,180,150$, and 1300). For each position the percentages of the maximal isometric force were calculated and participants were asked to maintain the respective force level for $5 \mathrm{~s}$. Electromyographic (EMG) activation of the Flexor Carpi Ulnaris and Extensor Digitorum muscles was recorded using bipolar surface electrodes. No significant differences were observed in maximal isometric strength between HS and $\mathrm{S}$ groups. Participants of HS group showed significantly $(P<0.05)$ smaller force coefficient of variability $(\mathrm{CV})$ and $\mathrm{SD}$ values at all submaximal levels of MVC at all wrist angles. The $\mathrm{CV}$ and SD values remained unaltered regardless of wrist angle. No difference in normalized agonist and antagonist EMG activity was observed between the two groups. It is concluded that long-term practice could be associated with decreased isometric force variability independently from muscular length and coactivation of the antagonist muscles.
\end{abstract}

Keywords Force variability · Isometric ·

Electromyography $\cdot$ Wrist flexors $\cdot$ Tennis $\cdot$ Handball

K. Salonikidis · I. G. Amiridis $(\square) \cdot$ N. Oxyzoglou .

E. S. S. de Villareal · A. Zafeiridis · E. Kellis

Laboratory of Neuromechanics,

Department of Physical Education and Sport Science at Serres,

Aristotle University of Thessaloniki, Thessaloniki, Greece

e-mail: jamoirid@phed.auth.gr

\section{Introduction}

The study of variability is essential to understand coordination. Muscle force fluctuations could depend on the presence or not of visual feedback (Christou 2005), the task specificity (Christou and Carlton 2001), the muscle group performing the task, the type and the intensity of the muscle contraction, and the physical activity status of the individual (Enoka et al. 2003). The relationship between the level of force and force variability remains controversial. Newell and Carlton (1988) reported that variability of force increases as level of force increases, while other data showed that variability increases linearly as the level of force increases up to $65 \%$ of maximum voluntary contraction (MVC), but then it decreases at force levels greater than 65\% of MVC (Sherwood and Smith 1980). In addition, it has been reported that at lower levels of force, variability of force increases at an increasing rate because of synchronization of the motor units (Yao et al. 2000). It has also been proposed that variability of force during isometric actions is described best by a sigmoidal, symmetric logistic function of the force signal (Christou et al. 2002).

In sports, well-established stereotyped trajectories are related to a greater accuracy of the movement and are often considered as the basis of steadiness in athletic performance. Trajectories with low levels of noise are preferentially chosen to maximize endpoint accuracy and to minimize endpoint variability during goal-directed movements (Hamilton and Wolpert 2002; Harris and Wolpert 1998). In this line, the application of steady isometric force from the wrist flexors to the tennis racket or to the ball in team sports such as the handball and the basketball might be extremely crucial for the final performance.

Neural mechanisms that underlie the fluctuation around an average value during isometric actions, involve motor 
unit properties and non-uniform activation of different muscles (Enoka et al. 2003). However, the physiological mechanism behind the potential differences in force fluctuation between elite and novice athletes is not well characterized. Strong young adults showed a greater force variability of knee extensors, than normal-strength participants beyond $50 \%$ of MVC, suggesting that strong participants display greater motor unit discharge rates (Christou et al. 2002). Moreover, it has been shown that even though strongest subjects could produce twice the peak force of the weakest subjects, there were no significant differences in peak force variability between the two groups (Carlton et al. 1993). Besides, the implementation of a simple finger task was accompanied by a reduction in the discharge rate variability of motor units, a decrease in the fluctuations of motor output of a hand muscle, and an improvement in manual dexterity (Kornatz et al. 2005).

The effects of long participation in elite sports on motor performance variability are not well understood. Traininginduced strength improvement is accompanied by an enhancement of motor-unit synchronization in the agonist muscles (Milner-Brown et al. 1973), as well as a reduction of the coactivation of the antagonists (Pousson et al. 1999). At the same time, however, training-induced technique improvement seems to be associated with an "independency" in the motor unit firing rate, lower motor unit synchronization, and substantially lower common drive (Semmler and Nordstrom 1998). Therefore, it could be interesting to study the physiological mechanism responsible for potential differences in force variability at the same force level between adults with different levels of expertise.

To our knowledge, very little information is available on potential differences in force variability between highly skilled and sedentary individuals. In addition, force fluctuations at different muscle lengths have not been previously investigated. The aim of this study was to examine whether the ability to sustain a constant application of submaximal strength is dependent on the level of expertise in young adults. The isometric force steadiness was studied over a wide range of angles, taking the force-length relationship of the particular muscles into consideration.

\section{Methods}

\section{Participants}

Twenty males were assigned, into one of two groups, the highly skilled group (HS, $n=10$, age: $22.0 \pm 0.8$ years, height: $179.9 \pm 4.4 \mathrm{~cm}$, mass: $79.1 \pm 7.4 \mathrm{~kg}$ ) and the sedentary group ( $\mathrm{S}, n=10$, age: $21.2 \pm 1.5$ years, height: $181.7 \pm 4.8 \mathrm{~cm}$, mass: $80.6 \pm 6.2 \mathrm{~kg}$ ). The main selection criteria were training experience and level of performance in a sport where forearm movement and muscle function are very important elements of performance. For this reason, tennis and handball players were preferred to form the HS group. Particularly, participants were included in the HS group when (a) they had a minimum 6 years training experience and a training frequency of more than five times a week; (b) they were active professional athletes in their sport. Based on these criteria, six participants were professional tennis players (official national ranking until No. 10) and the other four were professional handball players (first and second division National League). The participants of the $\mathrm{S}$ group were physical education students, active but without any participation to a particular strength and training program over the last 12 months. None of the participants had previous experience with this specific isometric task in order to avoid any learning effect. All participants were right-handed and free from any neurological or musculoskeletal impairment or disease. The experimental procedure was explained and prior to their inclusion in the study, the participants signed an informed consent form. Approval for the experiment was obtained from the institutional ethics committee on human research in accordance with the declaration of Helsinki.

\section{Strength measurements}

After a standardized warm-up, maximal and submaximal isometric data during wrist flexion at five different angular positions (230, 210, 180: anatomical zero, 150 and $\left.130^{\circ}\right)$ were collected using a $\mathrm{Kin} \mathrm{Com}^{\circledR}$ (Chattannooga, USA) isokinetic dynamometer. Participants were familiarized with the apparatus over a period of 1 week (three sessions of $40 \mathrm{~min}$ ) and they were also advised to refrain from any kind of physical activity for $48 \mathrm{~h}$ prior to testing. All tests were conducted over the same day period (15:30-18:00) to avoid any chronobiological effect. The rotation axis of the lever arm and wrist joint were adjusted and positioned according to the Kin Com System Manual. The right upper arm was restrained by two velcro straps and the forearm was strapped on a special manipulandum, which allowed to secure the elbow joint at $120^{\circ}$. The wrist joint remained free, the hand was positioned in vertical position and the fingers clasped the lever arm. Participants performed three isometric maximum voluntary contractions (MVC, duration $5 \mathrm{~s}$ ), in each angular position, randomly presented, and the average mean was used for the calculation of force levels. Following MVC assessment, the participants performed submaximal actions at 5, 10, 20, 50, and 75\% of MVC. Particularly, the participants were asked to develop and maintain, as stable as possible, the respective force level for $5 \mathrm{~s}$. Two efforts were performed at each level of MVC. Visual feedback of the exerted force during the isometric task was provided on-line both arithmetically and as a large bar 
histogram on the computer screen which was placed in front of the subject at eye level. First, the participants were informed about the type of visual feedback they were about to receive. They were then asked to watch the computer screen during contraction. The predetermined (target) level of force (for example 20\% MVC) was marked on the screen by the experimenter and it was visible for both the examiner and the participant. Consequently, upon initiation of the contraction, the participants could watch their recorded force which was displayed as a continuous rising bar histogram on the computer screen. When the target force level was reached, the subject was instructed to maintain the level of effort at this level for a period until the $5 \mathrm{~s}$ duration is completed. A 2-3 min rest was allowed between trials and the best effort was analyzed.

\section{Electromyography (EMG)}

A TEL100D (Biopac Systems, Inc., Goleta, CA) system including shielded electrode lead assemblies (Model SS2, Biopac Systems, Inc., Goleta, CA, bipolar silver/silver chloride electrodes, center-to-center interelectrode distance $=$ $2 \mathrm{~cm}$ ) interfaced to a portable amplifier/transmitter (Model TEL100M, Biopac Systems, Inc., Goleta, CA, CMRR > 110 $\mathrm{db}$ at $50 / 60 \mathrm{~Hz}$, bandwidth $=10-500 \mathrm{~Hz}$ ) was used to record the EMG activity of Flexor Carpi Ulnaris (FCU) m. and Extensor Digitorum (ED) muscles. Electrodes were attached over the palpable bellies of the FCU (at $1 / 4$ of the line between the medial epicondyle of the humerus and the pisiform bone) and ED muscles (at 1/4 of the line between the lateral epicondyle of the humerus and the midpoint of the line connecting the styloid process of the ulna and the radius) (Zipp 1982). Both Kin Com and EMG systems were interfaced to a Biopac MP100 Data Acquisition unit (Biopac Systems, Inc., Goleta, CA) sampling at $1024 \mathrm{~Hz}$. Following data collection, the EMG signals were full-wave rectified. Low impedance $(Z<500 \Omega)$ at the skin-electrode interface was obtained by shaving, abrading the skin, and cleaning it with alcohol. Special caution was given to the standardization of the experimental procedure (electrode position), to reduce the susceptibility of EMG to cross-talk (Kellis 1998). Signals were band-pass filtered (cut off: $10-330 \mathrm{~Hz}$ ) and digitally sampled at a frequency of $1000 \mathrm{~Hz}$ (full-wave rectification, fourth-order, zero-lag Butterworth, cut off: $100 \mathrm{~Hz}$ ). Power line hum at $50 \mathrm{~Hz}$ was also removed using a notch filter $(50 \mathrm{db}$ rejection at $50 \mathrm{~Hz})$. Integrated EMG activity over the $3 \mathrm{~s}$ of each isometric submaximal wrist flexion was expressed as a normalized value of the EMG during MVC.

\section{Data analysis}

The first and the last second were excluded from the analysis to avoid the transient phases in force development. For the remaining time interval from 1 to $4 \mathrm{~s}$, force fluctuations were quantified by calculating the SD of force and the coefficient of variability $(\mathrm{CV}=(\mathrm{SD} /$ mean $) \times 100)$. All EMG data collected were analyzed off-line using the AcqKnowledge $^{\circledR}$ (3.7.3, Biopac Systems, Inc., Goleta, CA) software. The integral of EMG activity over the $3 \mathrm{~s}$ of submaximal isometric action was expressed as a normalized value of the EMG activity observed during MVC. Maximal wrist extension was also performed at each specific angle and the recorded ED m (agonist) EMG was used to normalize the EMG of the same muscle when acting as antagonist.

\section{Statistical analysis}

All data are presented as means \pm SD. The SPSS 16.0 statistical package was used to analyze all data. A mixed 2 (group) $\times 5$ (level) $\times 5$ (angular position) analysis of variance (ANOVA) with repeated measures on force level and angular position was performed. All significant main effects were followed by Tukey post hoc tests. The level of significance was set at $P<0.05$.

\section{Results}

Maximal isometric force-angular position relationship

The MVC force of the HS group ranged from $26.19 \pm 6.48$ to $35.49 \pm 14.18 \mathrm{Nm}$ and in the $\mathrm{S}$ group from $24.85 \pm 5.93$ to $29.36 \pm 6.45 \mathrm{Nm}$. There were no significant $(P>0.05)$ differences in MVC force between highly skilled and sedentary individuals in all angular positions of wrist joint. Table 1 presents the moment-angular position relationship for highly skilled and sedentary individuals.

Table 1 Maximal isometric moments ( $\mathrm{Nm}$, mean values $\pm \mathrm{SD})$ at five angular positions of the wrist in sedentary and highly skilled individuals

\begin{tabular}{lll}
\hline & $\begin{array}{l}\text { Sedentary } \\
(n=10)\end{array}$ & $\begin{array}{l}\text { Highly skilled } \\
(n=10)\end{array}$ \\
\hline $230^{\circ}$ & $29.36 \pm 6.45$ & $35.49 \pm 14.18$ \\
$210^{\circ}$ & $26.11 \pm 5.67$ & $30.53 \pm 12.38$ \\
$180^{\circ}$ & $24.85 \pm 5.93$ & $26.19 \pm 6.48$ \\
$150^{\circ}$ & $26.04 \pm 3.58$ & $27.68 \pm 4.64$ \\
$130^{\circ}$ & $26.74 \pm 4.42$ & $29.51 \pm 4.71$ \\
\hline
\end{tabular}

Moment was similar between highly skilled and sedentary individuals across all angle levels (none-significant main effect of group). Furthermore, the pattern of change in moment across the levels of angle was similar between the two groups (none-significant group $\times$ angle interaction) 
Fig. 1 Representative submaximal isometric constant-force recordings (above) and EMG activation (below) of the agonist (Flexor Carpi Ulnaris, FCU) and antagonist (Extensor Digitorum, ED) muscles during steadiness task at 5, 10, 20, 50, and $75 \%$ of maximal voluntary contraction from a highly skilled (left) and a sedentary (right) participant
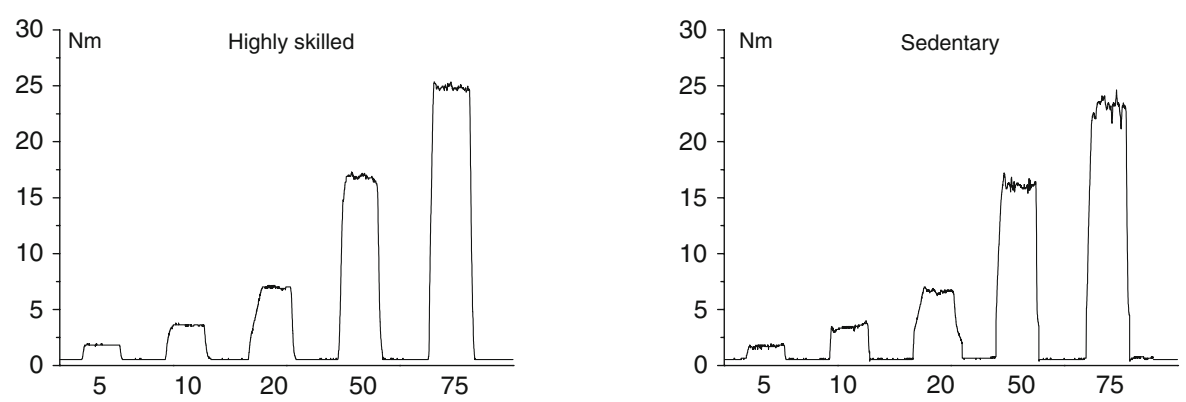

\section{$\%$ MVC}

5

FCU

10

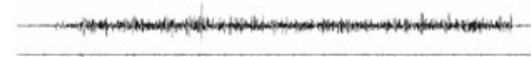

20



50
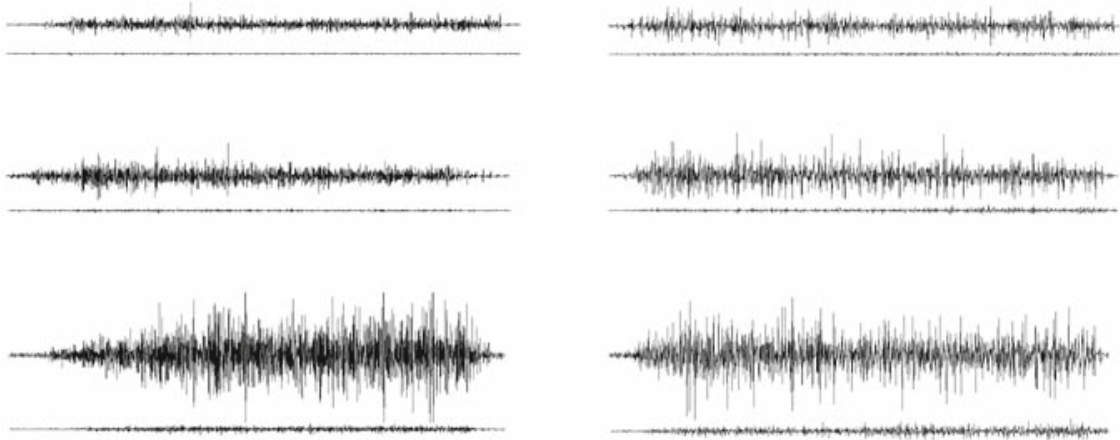

75
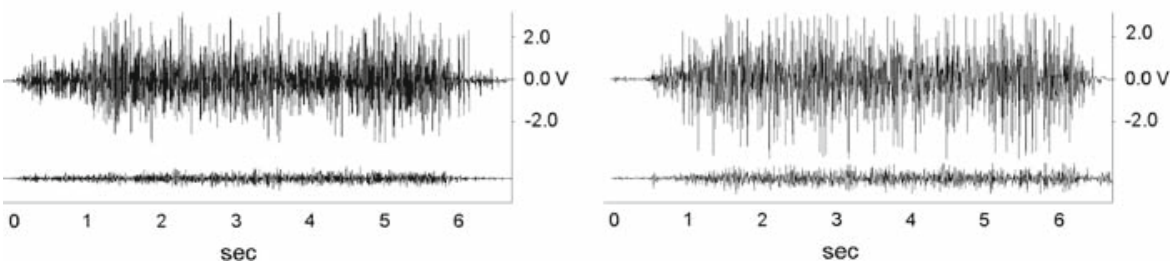

Force variability

ANOVA revealed a significant main effect of group $\left(F_{1,18}=167.08 ; P<0.001\right)$ on $\mathrm{CV}$ of force. Sedentary subjects demonstrated greater force fluctuation compared to highly skilled subjects irrespective of angle and intensity $(P<0.001)$, as suggested by no significant interactions of group by angle or/and intensity. Figure 1 presents row data from a highly skilled and a sedentary participant performing the submaximal isometric task. In general, the $\mathrm{CV}$ of force was higher at lower intensities (5 and 10\%) compared to higher intensities (50 and $75 \%)(P<0.05)$. Figure 2 provides the coefficient of variation of force in highly skilled and sedentary participants within each angle $(230,210,180,150$, and $\left.130^{\circ}\right)$ across 5 intensities $(5,10,20,50$, and $75 \%$ of MVC).
EMG recordings

The average \pm SD values for normalized iEMG across groups, angular positions, and intensities for both agonist and antagonist muscles during wrist flexion are presented in Table 2.

There was a significant main effect of intensity on normalized iEMG of the agonist muscles during wrist flexion $\left(F_{4,72}=96.628 ; P<0.001\right)$. As expected, the normalized iEMG gradually increased with increase in intensity from 5 to $75 \%$ of target force. However, this effect was similar between highly skilled and untrained individuals and was not affected by the angular position of the wrist during the constant-force tasks.

For the co-activation of the antagonist muscle during wrist flexion, the ANOVA showed a significant angle $x$ intensity interaction $\left(F_{20,360}=1.882 ; P<0.05\right)$ indicating 
Fig. 2 Coefficients of variation in highly skilled and sedentary individuals at five angular positions of the wrist joint. Highly skilled individuals showed lower fluctuations in target moment versus sedentary individuals across all intensities and angle levels (significant main effect of group, $P<0.001$; and none-significant interactions of group or/ and intensity or/and angle)
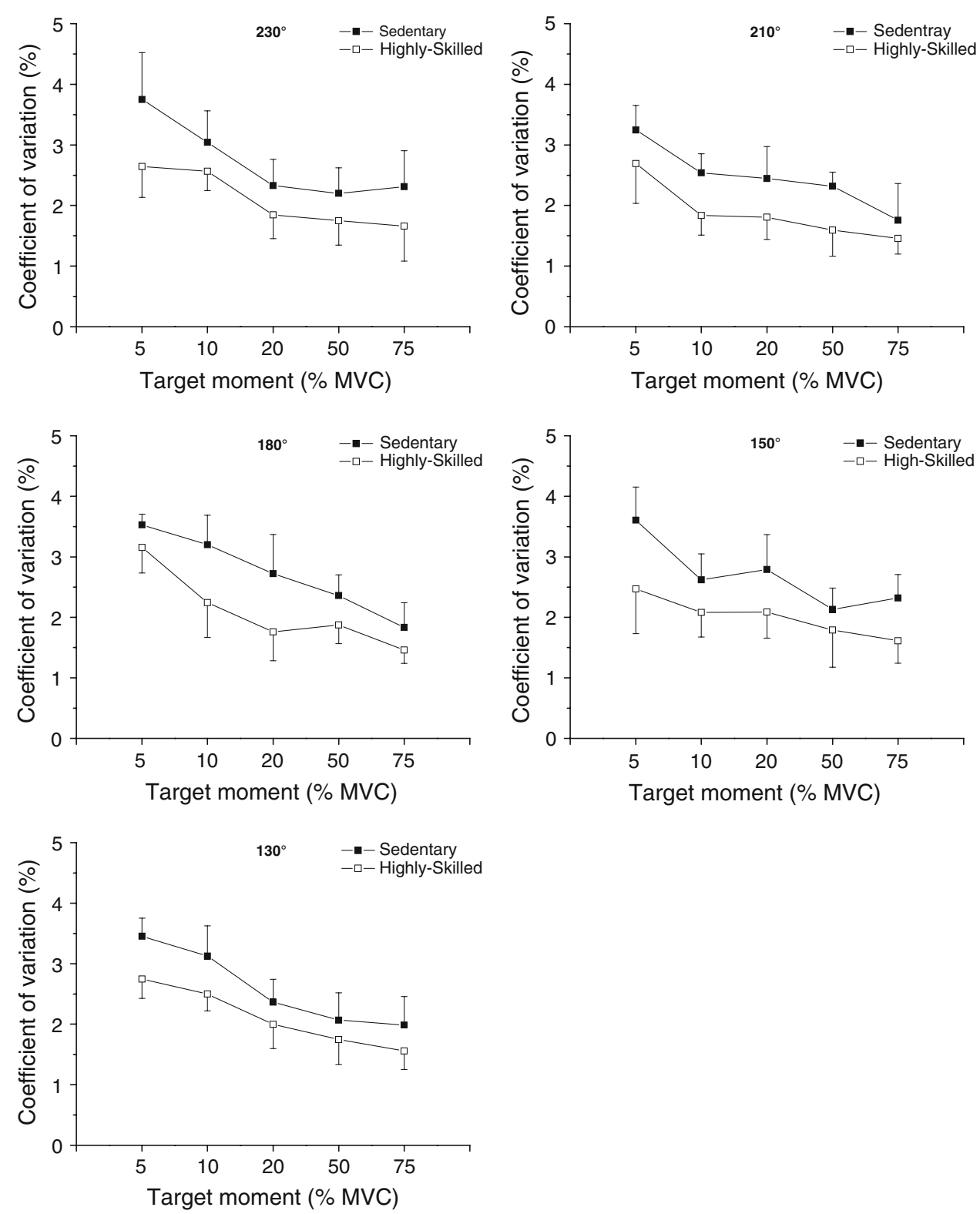

that the effect of task intensity on normalized iEMG was dependent on the angular position of the wrist joint. Similar to the agonist muscles, the pattern of increase in normalized iEMG of the antagonist muscles as force level increased was not significantly different between highly skilled and untrained individuals across five angular positions and six intensities $(P>0.05)$.

\section{Discussion}

The main finding of this study was that highly skilled individuals present a greater ability to perform steady submaximal isometric wrist flexions at matched levels of MVC than less skilled participants. This greater steadiness was not accompanied by group differences in the agonist and antagonist EMG and it was not length specific.

Experts are much steadier than similarly strong sedentary

Several hypotheses could be formulated to justify why experts are more accurate than sedentary young adults with the same level of force such as group differences in activation strategy of the agonist muscle, simultaneous activation by other synergistic muscles, antagonist coactivation, integration of visual feedback, and muscle fiber typology and mechanics. First, force fluctuations are dependent on the interaction between firing patterns of motor units of the agonist muscles (variability, firing rate, common modulation, and synchronization) (Jones et al. 2002; Kornatz et al. 
Table 2 Mean values \pm SD for normalized EMG (\%) for agonist and antagonist muscles of wrist joint at five different angular positions and five target moments

The pattern of increase in normalized EMG in agonist and antagonist muscles was similar between highly skilled and sedentary individuals across all angular positions and target moments (none-significant interactions of group or/and intensity or/and angle)

\begin{tabular}{|c|c|c|c|c|c|}
\hline & & \multicolumn{2}{|c|}{ Sedentary $(n=10)$} & \multicolumn{2}{|c|}{ Highly skilled $(n=10)$} \\
\hline & & Agonist & Antagonist & Agonist & Antagonist \\
\hline \multirow[t]{5}{*}{$230^{\circ}$} & $5 \%$ & $2.51 \pm 2.81$ & $3.04 \pm 2.80$ & $3.86 \pm 2.83$ & $3.73 \pm 2.05$ \\
\hline & $10 \%$ & $6.27 \pm 4.65$ & $4.59 \pm 3.80$ & $10.39 \pm 7.48$ & $4.15 \pm 3.86$ \\
\hline & $20 \%$ & $18.43 \pm 9.55$ & $5.06 \pm 4.11$ & $18.18 \pm 14.60$ & $3.96 \pm 2.37$ \\
\hline & $50 \%$ & $53.49 \pm 19.43$ & $13.78 \pm 5.33$ & $50.53 \pm 26.94$ & $8.92 \pm 5.82$ \\
\hline & $75 \%$ & $79.30 \pm 41.54$ & $19.15 \pm 8.16$ & $82.67 \pm 42.51$ & $17.16 \pm 9.63$ \\
\hline \multirow[t]{5}{*}{$210^{\circ}$} & $5 \%$ & $2.95 \pm 2.32$ & $3.75 \pm 3.95$ & $3.31 \pm 2.36$ & $2.20 \pm 1.61$ \\
\hline & $10 \%$ & $7.03 \pm 4.32$ & $2.36 \pm 1.17$ & $8.15 \pm 4.03$ & $1.84 \pm 1.23$ \\
\hline & $20 \%$ & $18.74 \pm 11.29$ & $4.62 \pm 2.66$ & $17.53 \pm 9.13$ & $3.89 \pm 3.09$ \\
\hline & $50 \%$ & $60.37 \pm 33.07$ & $10.84 \pm 4.15$ & $48.02 \pm 18.70$ & $9.35 \pm 4.62$ \\
\hline & $75 \%$ & $81.48 \pm 45.26$ & $17.30 \pm 9.22$ & $73.92 \pm 33.52$ & $15.34 \pm 8.16$ \\
\hline \multirow[t]{5}{*}{$180^{\circ}$} & $5 \%$ & $3.71 \pm 2.62$ & $2.88 \pm 2.60$ & $3.54 \pm 1.58$ & $2.03 \pm 1.48$ \\
\hline & $10 \%$ & $7.19 \pm 3.26$ & $2.34 \pm 1.8$ & $7.38 \pm 2.99$ & $1.86 \pm 1.01$ \\
\hline & $20 \%$ & $23.56 \pm 18.89$ & $4.88 \pm 3.06$ & $13.46 \pm 4.39$ & $3.92 \pm 2.28$ \\
\hline & $50 \%$ & $60.99 \pm 46.15$ & $9.01 \pm 4.70$ & $43.56 \pm 14.64$ & $9.31 \pm 4.81$ \\
\hline & $75 \%$ & $84.31 \pm 48.17$ & $15.67 \pm 7.86$ & $74.83 \pm 32.23$ & $19.97 \pm 15.5$ \\
\hline \multirow[t]{5}{*}{$150^{\circ}$} & $5 \%$ & $4.00 \pm 1.72$ & $3.25 \pm 3.54$ & $6.48 \pm 4.84$ & $2.23 \pm 1.24$ \\
\hline & $10 \%$ & $7.64 \pm 2.69$ & $3.29 \pm 3.10$ & $9.31 \pm 4.45$ & $2.54 \pm 1.46$ \\
\hline & $20 \%$ & $19.67 \pm 13.91$ & $5.43 \pm 4.79$ & $16.32 \pm 6.53$ & $3.70 \pm 1.93$ \\
\hline & $50 \%$ & $50.49 \pm 18.07$ & $10.09 \pm 3.80$ & $49.54 \pm 19.82$ & $12.18 \pm 5.39$ \\
\hline & $75 \%$ & $67.84 \pm 28.37$ & $18.97 \pm 5.14$ & $78.15 \pm 30.20$ & $20.87 \pm 11.2$ \\
\hline \multirow[t]{5}{*}{$130^{\circ}$} & $5 \%$ & $5.71 \pm 2.39$ & $3.31 \pm 1.86$ & $6.98 \pm 3.98$ & $2.63 \pm 1.44$ \\
\hline & $10 \%$ & $10.01 \pm 5.74$ & $7.21 \pm 6.69$ & $11.28 \pm 6.13$ & $3.33 \pm 1.99$ \\
\hline & $20 \%$ & $22.29 \pm 12.07$ & $5.10 \pm 2.01$ & $18.67 \pm 7.69$ & $6.40 \pm 4.63$ \\
\hline & $50 \%$ & $56.00 \pm 22.01$ & $10.47 \pm 5.04$ & $54.02 \pm 19.57$ & $13.31 \pm 6.30$ \\
\hline & $75 \%$ & $77.41 \pm 24.07$ & $22.18 \pm 9.81$ & $76.26 \pm 33.10$ & $26.23 \pm 14.2$ \\
\hline
\end{tabular}

2005; Laidlaw et al. 2000; Moritz et al. 2005; Semmler and Nordstrom 1998; Taylor et al. 2003; Tracy et al. 2005; Vaillancourt and Newell 2003). A greater motor-output variability could be the result of an altered agonist activity. It has been reported that long-term learning of a motor task was associated with differences in higher centers and preprogrammed descending commands and adaptations in the contralateral somatosensory and motor cortex and the putamen (Floyer-Lea and Matthews 2005). Distinct functional changes in both primary somatosensory and motor cortex increase representation of the learned specific movement sequence and indicate the importance of afferent feedback to this form of predominantly implicit motor learning (Floyer-Lea and Matthews 2005). Motor-unit synchronization, as a result of strength training, could decrease the steadiness of the force exerted by the muscle in simulated contractions (Yao et al. 2000). Force tremor is reported to be higher in the strength-trained subject, median in the untrained subject and lower in the skill-trained subject (Semmler and Nordstrom 1998). However, it has recently been reported that strength training does not affect the accuracy of force gradation in an isometric task in young men, supporting that there is no evidence to expect a loss in accuracy as a result of strength training (Smits-Engelsman et al. 2008). In the same line, Hamilton et al. (2004), comparing force fluctuation in four upper limb muscles with widely varying maximal forces and numbers of motor units (elbow extensors, wrist flexors, first dorsal interosseus, and thumb extensors), reported that stronger muscles with greater numbers of motor units had the lowest fluctuations.

Second, one may consider that the torque measured about the wrist is the sum of all the forces acting on that joint. When more than one muscle is active, there are many finite combinations of synergist and antagonist forces that can produce a given torque about the joint. Therefore, fluctuations in joint torque reflect not only the summation of force fluctuations in individual muscles, but also the shape and the temporal association in the waveform of force fluctuations between muscles. According to Shinohara et al. (2009), an asynchronous compensatory fluctuation between individual muscles forces could happen due to subtle differences in the neural activation strategy, force production profiles, and/or force transmission characteristics across muscles. Third, a lower coactivation of the antagonists in 
highly skilled compared to sedentary individuals is clearly demonstrated during maximal efforts (Amiridis et al. 1996). However, in our study, the greater steadiness exhibited by experts was not accompanied by alterations at the level of the antagonist coactivation (Table 2), confirming also previous reports (Burnett et al. 2000). Four, a greater integration of visual feedback by the elite individuals could maximize information transmission and minimize force fluctuations during constant isometric tasks (Christou 2005). Finally, differences in morphological (theoretically, type II fibers having higher fusing frequencies than type I fibers, will produce more tremor at a given recruitment level) (Yao et al. 2000) and mechanical properties (series compliance and frictional interactions among muscle fibers) (Troiani et al. 1999) could also be involved to a nonlinear summation and mechanical differences between highly skilled and sedentary individuals.

The absence of differences in wrist flexors MVC scores between athletes and sedentary individuals could be partially justified by the sport skill level, velocity specificity, and the type of action. It could be assumed that the welldocumented neural mechanism of motor unit synchronization following strength training (Milner-Brown et al. 1973) has not occurred in our experts. This is also supported by an unchanged coactivation level during maximal efforts, which almost always accompanies strength training adaptations (Jubeau et al. 2006).

Steadiness increases as the \% MVC force increases

In the present study, the quantification of variability was based on the use of relative \% MVC target levels and not absolute levels of force, as suggested previously for a generalized description of force variability during isometric actions (Christou et al. 2002). Our results showed clearly that as the force percentage increased, the $\mathrm{CV}$ of force decreased, regardless of the expertness level (Fig. 2). This seems to be in line with previous studies (Christou and Carlton 2002, Laidlaw et al. 2000), but not with others which reported that, during isometric handgrip, force tremor amplitude increased from 20 to $60 \%$ MVC and decreased at 80\% MVC (Loscher and Gallash 1993). However, the pattern of the variability alteration could be described better as a sigmoidal (Christou et al. 2002; Loscher and Gallash 1993) than an exponential (Slifkin and Newell 1999) relationship. Differences in temporal parameters, age of participants, sex, type of action, muscular group, and visual feedback could only partly explain such a discrepancy. We propose that at very low levels of force both experts and active young adults present an increased variability because of synchronization of motor units. When greater levels of force are exerted, the variability decreases. Although in our study we did not examine motor unit firing patterns, it is tempting to consider that a more stochastically independent discharge of motor units in experts contributed to the lower force fluctuations in these participants compared with sedentary ones (Semmler and Nordstrom 1998; Christakos 1982).

Steadiness remains unaffected from the muscular length

No wrist angle effect on force variability measurements was observed in both experimental groups, suggesting that the length of the flexor muscles does not influence the steady application of force. The absence of any length specificity could support that expert and sedentary participants used their wrist flexors across the whole range of motion in a similar manner. The maximum force was attained when the wrist was maximally extended (Table 1 ). This confirms a previous study which combined morphological data from a cadaver with a mathematical model (Friden and Lieber 1998).

In conclusion, we observed that a group of individuals who have a longer expertise in utilizing the wrist muscles present significant differences on force variability compared with individuals with no previous experience. However, this observation was not accompanied by group differences in either agonist or antagonist muscle activity patterns. Because extended practice increases the importance of task-specific sources of variation and defines someone as an expert in one task but novice to another, further examination of other factors, such as motor unit behavior is required to examine whether force variability is related to skill level.

Open Access This article is distributed under the terms of the Creative Commons Attribution Noncommercial License which permits any noncommercial use, distribution, and reproduction in any medium, provided the original author(s) and source are credited.

\section{References}

Amiridis IG, Martin A, Morlon B, Martin L, Cometti G, Pousson M, van Hoecke J (1996) Co-activation and tension-regulating phenomena during isokinetic knee extension in sedentary and highly skilled humans. Eur J Appl Physiol 73:149-156

Burnett RA, Laidlaw DH, Enoka RM (2000) Coactivation of the antagonist muscle does not covary with steadiness in old adults. J Appl Physiol 89:61

Carlton LG, Kim KH, Liu YT, Newell KM (1993) Impulse variability in isometric tasks. J Mot Behav 25:33-43

Christakos CN (1982) A study of the muscle force waveform using a population stochastic model of skeletal muscle. Biol Cybern 44:91-106

Christou EA (2005) Visual feedback attenuates force fluctuations induced by a stressor. Med Sci Sports Exerc 37:2126-2133

Christou EA, Carlton LG (2001) Old adults exhibit greater motor output variability than young adults only during rapid discrete isometric contractions. J Gerontol A Biol Sci Med Sci 56:B524-532 
Christou EA, Carlton LG (2002) Age and contraction type influence motor output variability in rapid discrete tasks. J Appl Physiol 93:489-498

Christou EA, Grossman M, Carlton LG (2002) Modeling variability of force during isometric contractions of the quadriceps femoris. J Mot Behav 34:67-81

Enoka RM, Christou EA, Hunter SK, Kornatz KW, Semmler JG, Taylor AM, Tracy BL (2003) Mechanisms that contribute to differences in motor performance between young and old adults. J Electromyogr Kinesiol 13:1-12

Floyer-Lea A, Matthews PM (2005) Distinguishable brain activation networks for short- and long-term motor skill learning. J Neurophysiol 94:512-518

Friden J, Lieber RL (1998) Evidence for muscle attachment at relatively long lengths in tendon transfer surgery. J Hand Surg 23:105-110

Hamilton AF, Wolpert DM (2002) Controlling the statistics of action: obstacle avoidance. J Neurophysiol 87:2434-2440

Hamilton AF, Jones KE, Wolpert DM (2004) The scaling of motor noise with muscle strength and motor unit number in humans. Exp Brain Res 157:417-430

Harris CM, Wolpert DM (1998) Signal-dependent noise determines motor planning. Nature 20:780-784

Jones KE, Hamilton AF, Wolpert DM (2002) Sources of signal-dependent noise during isometric force production. J Neurophysiol 88:1533-1544

Jubeau M, Zory R, Gondin J, Martin A, Maffiuletti N (2006) Late neural adaptations to electrostimulation resistance training of the plantar flexors muscles. Eur J Appl Physiol 98:202-211

Kellis E (1998) Quantification of quadriceps and hamstring antagonist activity. Sports Med 25:37-62

Kornatz KW, Christou EA, Enoka RM (2005) Practice reduces motor unit discharge variability in a hand muscle and improves manual dexterity in old adults. J Appl Physiol 98:2072-2080

Laidlaw DH, Bilodeau M, Enoka RM (2000) Steadiness is reduced and motor unit discharge is more variable in old adults. Muscle Nerve 23:600-612

Loscher WN, Gallash E (1993) Myo-electric signals from two extrinsic hand muscles and force tremor during isometric handgrip. Eur $\mathbf{J}$ Appl Physiol 67:99-105
Milner-Brown HS, Stein RB, Yemm R (1973) The orderly recruitment of human motor units during voluntary isometric contractions. J Physiol 230:359-370

Moritz CT, Barry BK, Pascoe MA, Enoka RM (2005) Discharge rate variability influences the variation in force fluctuations across the working range of a hand muscle. J Neurophysiol 93:2449-2459

Newell KM, Carlton LG (1988) Force variability in isometric responcws. J Exp Psychol Hum Percept Perform 14:37-44

Pousson M, Amiridis IG, Cometti G, Van Hoecke J (1999) Velocity specific training in elbow flexors. Eur J Appl Physiol 80:367-372

Semmler JG, Nordstrom MA (1998) Motor unit discharge and force tremor in skill- and strength-trained individuals. Exp Brain Res 119:27-38

Sherwood DE, Smith RA (1980) The relationship between force and force variability in minimal and near-maximal static and dynamic contractions. J Mot Behav 12:75-89

Shinohara M, Yoshitake Y, Kouzaki M (2009) Alterations in synergistic muscle activation impact fluctuations in net force. Med Sci Sports Exerc 41:191-197

Slifkin AB, Newell KM (1999) Noise, information transmission, and force variability. J Exp Psychol Hum Percept Perform 25:837-851

Smits-Engelsman B, Smits R, Oomen J, Duysens J (2008) Strength training does not affect the accuracy of force gradation in an isometric task in young men. Int J Sports Med 29:59-65

Taylor AM, Christou EA, Enoka RM (2003) Multiple features of motor-unit activity influence force fluctuations during isometric contractions. J Neurophysiol 90:1350-1361

Tracy BL, Maluf KS, Stephenson JL, Hunter SK, Enoka RM (2005) Variability of motor unit discharge and force fluctuations across a range of muscle forces in older adults. Muscle Nerve 32:533-540

Troiani D, Filippi GM, Bassi FA (1999) Nonlinear tension summation of different combinations of motor units in the anesthetized cat peroneus longus muscle. J Neurophysiol 81:771-780

Vaillancourt DE, Newell KM (2003) Aging and the time and frequency structure of force output variability. J Appl Physiol 94:903-912

Yao W, Fuglevand AJ, Enoka RM (2000) Motor-unit synchronization increases EMG amplitude and decreases force steadiness of simulated contractions. J Neurophysiol 83:441-452

Zipp P (1982) Recommendations for the standardization of lead positions in surface electromyography. Eur J Appl Physiol 50:41-54 